\title{
SEDOPTICA Newsletters
}

\section{Noticias de SEDOPTICA}

Continuando el espíritu de esta sección de noticias que iniciamos en el número pasado de OPA, recogemos a continuación las actividades de SEDOPTICA en el periodo de julio a septiembre.

\section{Congresos y Jornadas}

Son varios los acontecimientos que tuvieron lugar en este trimestre de verano. A final del mes de julio se celebraron dos de los congresos propios de nuestra sociedad. En los días del 19 al 22 de julio se celebró en Ourense el XI Congreso Nacional del Color, organizado por el Comité de Color, siendo Humberto Michinel el presidente del Comité Organizador. Por otro lado, entre los días 20 y 22 de julio se celebró en Alicante la XXV Reunión Nacional de Espectroscopia - IX Congreso Ibérico de Espectroscopia, organizado por el Comité de Espectroscopia en colaboración con otras sociedades, siendo Antonio Canals presiente del Comité Organizador. Ambos congresos tuvieron una importante participación y permanecen como referentes nacionales en sus respectivos ámbitos. Igualmente, en el marco del Congreso Nacional de Color se celebró en Ourense el III Encuentro de Jóvenes Investigadores de Óptica (EJIO) organizado por el Área Joven de SEDOPTICA.

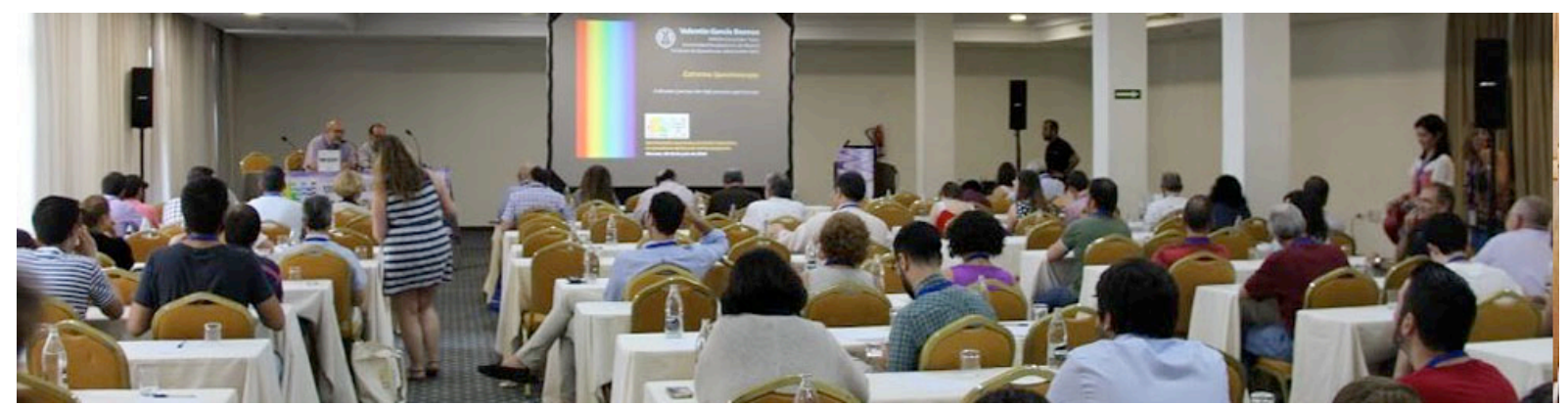

Fig. 1. Fotografía de una sesión de la XXV Reunión Nacional de Espectroscopia - IX Congreso Ibérico de Espectroscopia.

En ambos congresos se procedió a la renovación de los cargos directivos de ambos comités. La renovación del Comité de Color será efectiva en enero de 2017, cuando pasará a estar presidido por Juan Luis Nieves, con Meritxell Vilaseca como vicepresidenta, y Esther Perales como secretaria. El Comité de Espectroscopia ha pasado a estar presidido por la María Rosa López, siendo Paz Sevilla vicepresidenta, y María de la Vega Cañamares secretaria. Deseamos desde aquí agradecer a las nuevas directivas por su implicación en SEDOPTICA, y los ahora presidentes salientes, Francisco Heredia en el Comité de Color, y Belén Maté en el Comité de Espectroscopia, por el excelente trabajo realizado.

SEDOPTICA apoyó también la celebración en Barcelona del $15^{\text {th }}$ Workshop on Information Optics WIO2016, celebrado en Barcelona entres los días 11 y 15 de julio, organizado por Artur Carnicer. Igualmente, SEDOPTICA apoyó la International School on Light Sciences and Technologies - ISLiST celebrada en Santander del 20 al 24 de junio, y dirigida por José Miguel López-Higuera. En dicha Escuela, se hizo entrega de la $5^{a}$ edición del Premio Justiniano Casas patrocinado por la empresa INFAIMON. La Fig. 2 muestra una fotografía del acto, con la presencia de Alba Peinado, galardonada con el primer premio por la Tesis Doctoral "Design of polarimeters based on liquid crystals and biaxial crystals for polarization metrology", dirigida por Juan Campos y Angel Lizana en la Universidad Autónoma de Barcelona, y de Alejandro Cámara, accésit por la Tesis Doctoral titulada "Optical beam characterisation via phase-space tomography", dirigida en la Universidad Complutense de Madrid por Tatiana Aliyeva y María Luisa Calvo. Desde aquí nuestra enhorabuena a los galardonados, y al Comité de Técnicas de la Imagen, representado por su presidente Jesús Lancis, y su secretaria, Inmaculada Pascual, por esta importante actividad. 


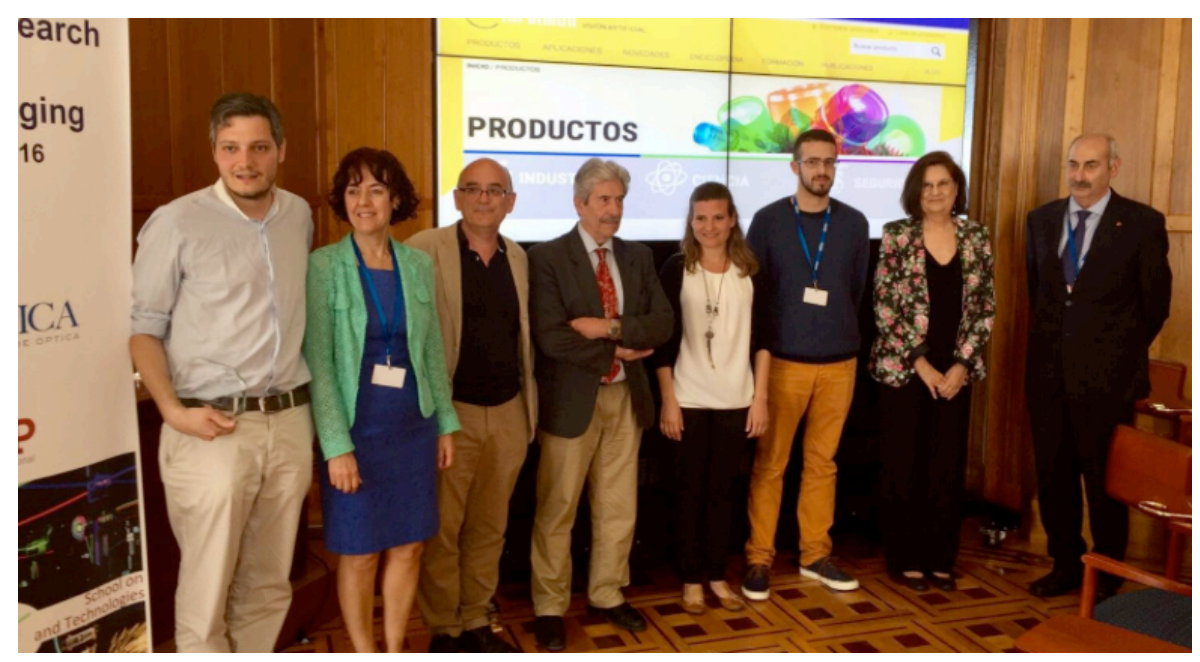

Fig. 2. Acto de entrega del Premio Justiniano Casas de Imagen Óptica. De izquierda a derecha Angel Lizana, Inmaculada Pascual, Jesús Lancis, Santiago Vallmitjana, Alba Peinado, José Manuel Soto (en representación de Alejandro Cámara), María Luisa Calvo y José Miguel López Higuera.

Igualmente, SEDOPTICA apoyó la celebración en Barcelona del $15^{\text {th }}$ Workshop on Information Optics WIO2016, celebrado en Barcelona entres los días 11 y 15 de julio, organizado por Artur Carnicer. Dicho congreso incluyó la exposición "Investigadoras en la Luz y en las Tecnologías de la Luz", y una presentación relacionada a cargo de María Sagrario Millán (Fig. 3).

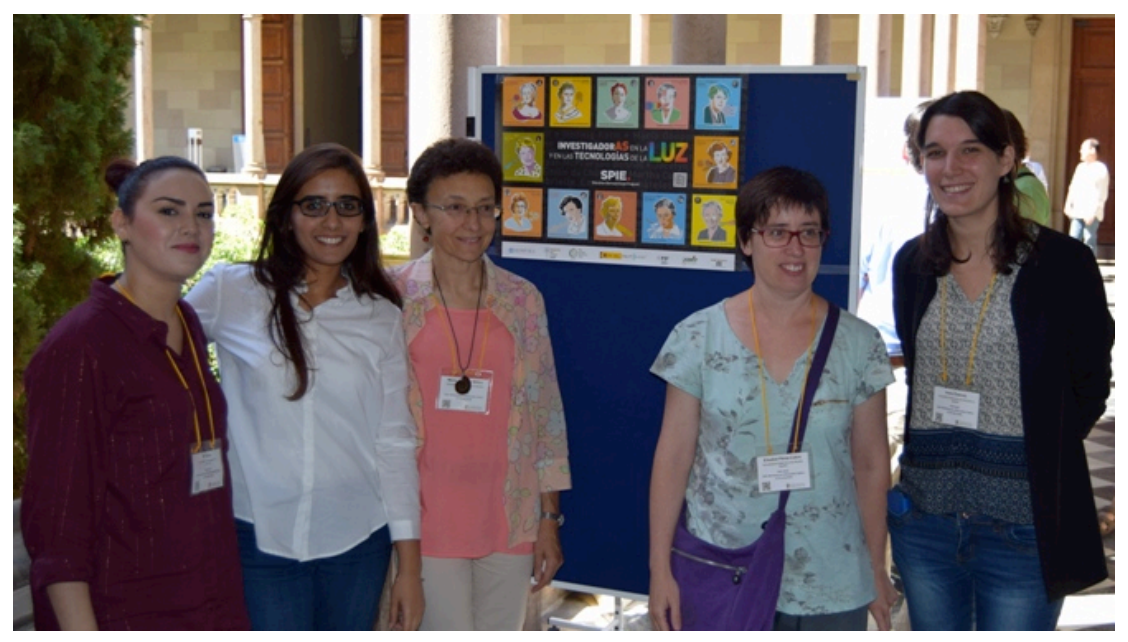

Fig. 3. Exposición “Investigadoras en la Luz y en las Tecnologías de la Luz” en el congreso WIO2016. De izquierda a derecha: Merim

Aprovechamos para recordar otras actividades internacionales que apoyamos desde SEDOPTICA, y que se celebrarán próximamente, como son la IX Reunión Iberoamericana de Óptica y XII Reunión Iberoamericana de Óptica, Láseres y Aplicaciones (RIAO/OPTILAS), que se celebrará en el próximo mes de noviembre en Pucón (Chile), o la III International Conference on Applications of Optics and Photonics AOP2017, que se celebrará en Faro (Portugal) en Mayo de 2017. En ambos casos, las contribuciones presentadas al congreso podrán directamente proponerse también en forma de artículos a la revista OPA. Igualmente, consideramos de trascendencia el congreso Frontiers in Optics que celebra el centenario de OSA - Optical Society of America, y que se celebrará en Rochester del 17 al 21 de octubre.

\section{Socios de SEDOPTICA}

El día 12 de julio se celebró en la Universidad de Granada un acto homenaje a Enrique Hita, presidente de SEDOPTICA en el periodo 1999-2002. Dicho acto, presidido por la Rectora de la Universidad de Granada, 
contó con la representación de varios ex-presidentes de SEDOPTICA, y sirvió también de marco para la entrega del diploma y placa de Socio de Honor de SEDOPTICA.
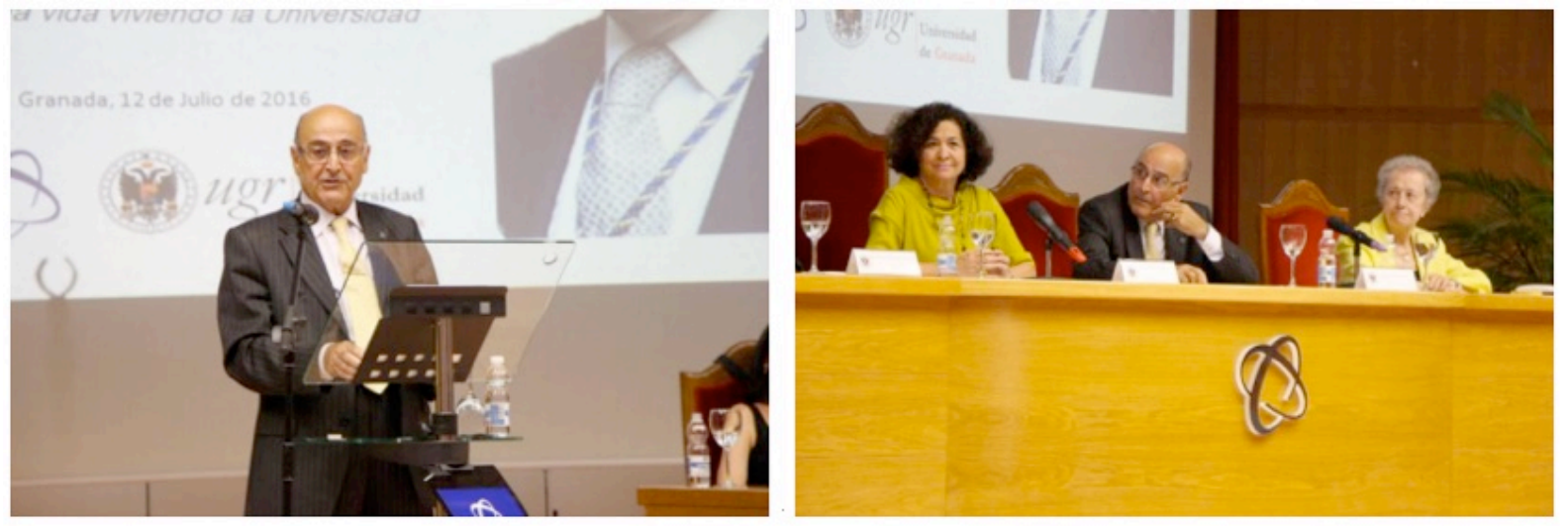

Fig. 3. Fotografías del acto homenaje al Prof. Enrique Hita.

Deseamos en este punto también destacar y felicitar a Carlos Ferreira por la concesión de la categoría de Fellow Member de EOS - European Optical Society. La ceremonia de entrega tendrá lugar en Berlín en el marco del congreso EOSAM 2016.

Asimismo, deseamos felicitar a María Sagrario Millán y a Pablo Aitor Postigo, por la reciente concesión de la categoría de Senior Member de OSA - Optical Society of America.

Por último, deseamos expresar nuestra gran tristeza por el reciente fallecimiento de nuestro socio de honor, el Prof. Felipe Mateos, el pasado día 5 de septiembre en Alicante. Felipe desarrolló una labor fundamental para SEDOPTICA durante el largo periodo en que ocupó el cargo de Editor de la revista OPA. Son muchas las muestras de pesar que hemos recibido por parte de muchos socios y que hemos transmitido a la familia.

\section{Colaboraciones, materiales, y recursos}

El día 12 de julio se firmó un acuerdo de colaboración entre SEDOPTICA y SECPho, con el objetivo de implementar formas de colaboración que ayuden a acortar la distancia que muchas veces se produce entre la academia y la industria. La fotografía muestra la firma del acuerdo, por parte de Luz Ruiz y Santiago Vallmitjana, respectivos presidentes. Un primer aspecto interesante es la colaboración en la difusión de la bolsa de trabajo de SECPho.

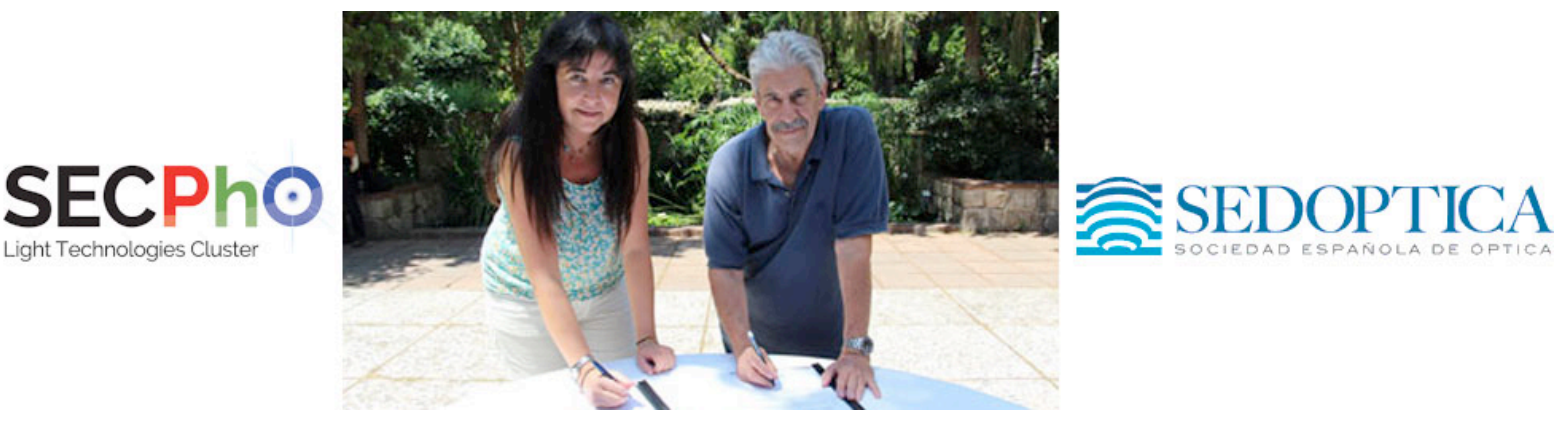

Fig. 3. Fotografía de la firma del acuerdo de colaboración SEDOPTICA-SECPho.

Deseamos de nuevo recordar la gran cantidad de recursos disponibles en la web del Año Internacional de la Luz en España www.luz2015.es, en cuyo apartado de recursos podréis encontrar numerosos vídeos, material gráfico, artículos de divulgación, podcasts, el juego educativo entreREDes, etc. También el blog para la difusión de actividades de divulgación de la óptica, en el cual se pueden notificar las actividades de divulgación de la óptica. 
Todo este material puede resultar de especial interés para la nueva iniciativa internacional difusión. denominada el Day of Photonics, a la cual os invitamos a cooperar organizando eventos el próxima día 21 de octubre. Desde SEDOPTICA recogeremos el conjunto de actividades que se realicen para darles una mayor

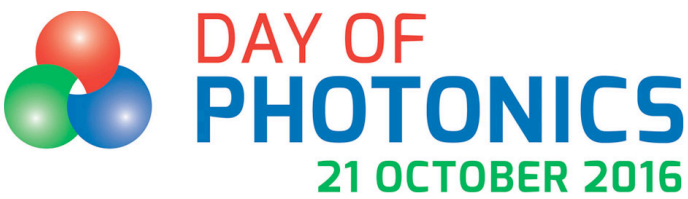

Barcelona, septiembre de 2016
Santiago Vallmitjana

Presidente de SEDOPTICA

Ignacio Moreno

Vicepresidente de SEDOPTICA

Joaquín Campos

Presidente saliente de SEDOPTICA

DOI: http://dx.doi.org/10.7149/OPA.49.3.iii 